\title{
Leitlinien-Update
}

\section{Akute Apoplextherapie ohne Altersbegrenzung}

Das Alter ist kein Hindernis mehr für eine rasche Öffnung verstopfter Hirngefäße: Auch für über 80 -Jährige Patienten werden inzwischen Lyse und Thrombektomie empfohlen.

Eigentlich haben sich Neurologen und Intensivmediziner schon in der Vergangenheit wenig um eine obere Altersbegrenzung für die Thrombolyse oder die Thrombektomie geschert, „weil solche Kontraindikationen nicht auf Daten beruhten, sondern auf Einschlusskriterien in randomisierten Studien“, sagt Prof. Dr. Hans-Christoph Diener vom Uniklinikum Essen im Gespräch mit „Springer Medizin“. Über 80-Jährige wurden in solchen Studien eben nicht aufgenommen.

Ähnliches gilt für andere Einschränkungen wie einen systolischen Blutdruck über $140 \mathrm{mmHg}$, einen vorbestehenden Schlaganfall oder Hypertonie und Diabetes. "Registerdaten haben gezeigt, dass die meisten Kontraindikationen keine sind“, berichtet Diener. Dies wurde nun in einem Update zur Schlaganfall-Akuttherapie-Leitlinie berücksichtigt.

\section{Empfehlungen zur Thrombektomie} Das Update ist jedoch vor allem nötig geworden, weil fünf Studien einen hohen Nutzen der mechanischen Thrombektomie mit modernen Stent-Retrievern zeigen konnten. Die wichtigsten Punkte:
- Eine Thrombektomie mit Stent-Retrievern wird primär bei einem distalen Verschluss der A. carotis interna oder einem proximalen Verschluss der A. cerebri media empfohlen, und zwar innerhalb von sechs Stunden nach Symptombeginn. Im 4,5-Stunden-Fenster sollten die Patienten zusätzlich eine systemische Lyse mit rtPA erhalten. Die Thrombektomie darf jedoch nicht die Einleitung der Lyse verzögern, umgekehrt sollte die Lyse nicht die Thrombektomie ausbremsen.

- Die Thrombektomie kann auch noch später als sechs Stunden nach dem Symptombeginn erwogen werden, z. B. dann, wenn die Mismatch-Bildgebung und Kollateralendarstellung Patienten mit Risikogewebe identifizieren.

- Für Krankenhäuser, die keine Möglichkeit zur Thrombektomie haben, wird ein „Drip and ship“-Konzept vorgeschlagen: Die Patienten erhalten möglichst schnell eine Lysetherapie (drip). Kommt eine Thrombektomie infrage, werden die betroffenen Patienten schnellstmöglich in eine Einrichtung mit Katheterbehandlung überführt (ship).

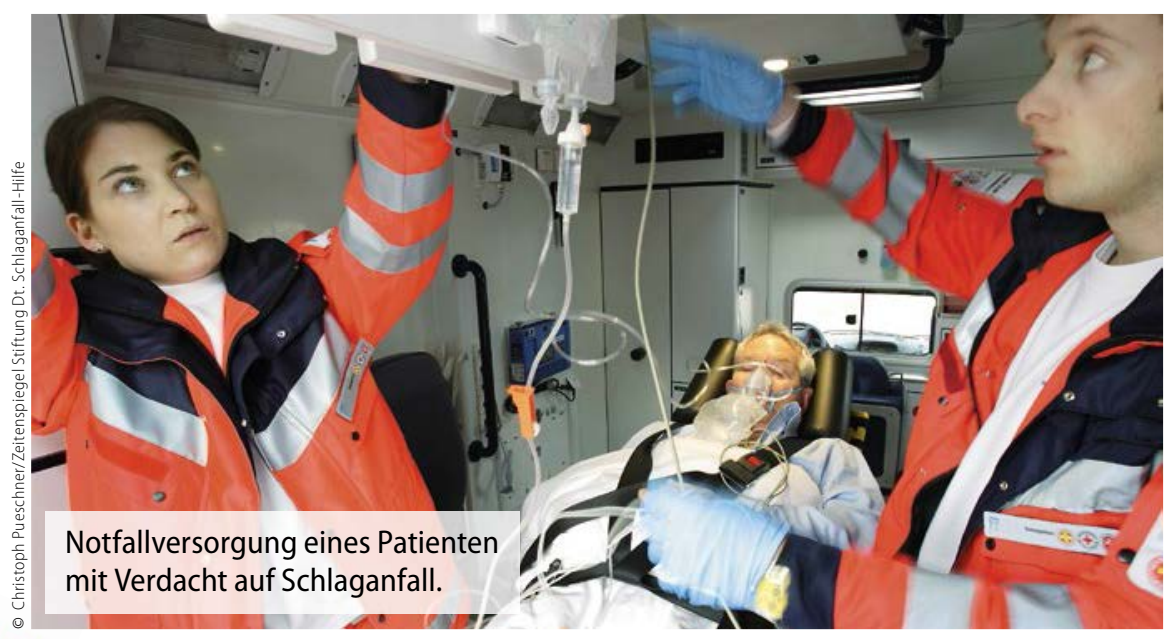

\section{Empfehlungen zur Thrombolyse}

Außerdem kommen einige Änderungen bei der Thrombolyse hinzu:

- Die Lyse mit rtPA wird innerhalb eines 4,5-Stunden-Fensters empfohlen. Das Fenster kann jedoch bei bestimmten Patienten auf 6 Stunden erweitert werden, vor allem wenn sich Risikogewebe identifizieren lässt.

- Ärzte können die Thrombolyse auch bei Patienten mit geringer Schlaganfallschwere, hohem Alter, rückläufigen Symptomen, mit Diabetes, Schlaganfall in der Anamnese, Schlaganfall oder größerer Operation in den letzten drei Monaten oder einem epileptischen Anfall erwägen. Sie sollten den Nutzen aber sorgfältig gegen Risiken wie Blutungen oder Ödeme abwägen.

- Unter Antikoagulanzien ist die Thrombolyse in Ausnahmefällen möglich. Bei Einnahme von VitaminK-Antagonisten gilt das Blutungsrisiko bei einem INR-Wert bis 1,7 als vertretbar. Unter einer Behandlung mit direkt wirksamen oralen Antikoagulanzien (DOAK) kann die Lyse nach Angaben der Leitlinie in Erwägung gezogen werden, wenn Gerinnungstests normal sind oder die Patienten bei normaler Nierenfunktion in den letzten 48 Stunden keine direkt wirksamen oralen Antikoagulanzien eingenommen haben.

Die mechanische Gefäßöffnung ist bei solchen Patienten hingegen weniger problematisch: „Man kann Patienten, die antikoaguliert sind, primär thrombektomieren“, berichtet Diener.
Update zur SchlaganfallAkuttherapie-Leitlinie:

Ringleb PA et al. Akuttherapie des ischämischen Schlaganfalls - Ergänzung 2015 - Rekanalisierende Therapie. AWMF-Nr.: 030/140 